\title{
Essai de prédétermination des débits d'étiage sur les cours d'eau présentant des pertes en région calcaire. L'exemple de la Tille entre Till-Chatel et Spoy (21)
}

\author{
An attempt to pre-determine low water flows \\ in streams losing water in calcareous regions: \\ example of the Tille river between Til-Chatel and Spoy (21)
}

\author{
M. Marion \\ Hydrogéologue, chargé d'études au S.R.A.E. Bourgogne
}

\section{Introduction}

Dans les régions calcaires la connaissance du régime des cours d'eau ne peut être appréhendée qu'au droit des stations de jaugeage. La transposition des caractéristiques principales du régime de la rivière soit à l'amont, soit à l'aval de la station, peut se traduire par des surestimations ou sous-estimations importantes suivant la répartition des apports ou des pertes entre la station prise en compte et la section où l'on désire estimer les débits.

Un tel problème se pose pour la prédétermination des débits d'étiage, notamment dans le cadre de la fixation de normes de rejet. II convient alors d'estimer au droit du site envisagé :

- les débits caractéristiques d'étiage (par exemple débit moyen minimal sur $n$ jours consécutifs pour différentes périodes de retour);

- la durée pendant laquelle la rivière peut s'assécher pour différentes périodes de retour (par exemple assèchement pendant 30 jours consécutifs 2 années sur 10).

Il est bien évident que seules des mesures et observations en nombre suffisant, et adaptées au problème posé, permettront d'estimer les débits au droit d'un site dépourvu de station de jaugeage, surtout si celui-ci se situe au niveau d'une zone de pertes où la rivière peut s'assécher.

De tels problèmes se posent sur la Tille, où différents projets de stations d'épuration sont envisagés, notamment à Is-sur-Tille et Til-Châtel.

\footnotetext{
* Hy drogéologue, chargé d'études au Service Régional de l'Aménagement des Eaux de Bourgogne - Dijon.
}

Cadre général du système karstique Tille-IgnonVenelle-Bèze

\section{Cadre géologique et circulations karstiques}

Le bassin karstique des sources de la Bèze est traversé dans sa partie ouest par trois rivières prenant naissance à l'extérieur de ce bassin : il s'agit de la Venelle, de la Tille et de son affluent l'Ignon (fig. 1).

Ces trois rivières sont pérennes à leur entrée dans le bassin karstique au niveau du "seuil" marneux de l'Oxfordien moyen à supérieur (faciès "Argovien"). Après la traversée de cet écran imperméable elles vont s'écouler sur un complexe calcaire récifal de l'Oxfordien supérieur (faciès "Rauracien") ainsi que sur les calcaires compacts du Kimméridgien inférieur (faciès "Séquanien"). Bien qu'ayant des faciès variés, ces formations calcaires présentent une même karstification et montrent un comportement identique vis à vis des circulations souterraines. Il n'existe pratiquement aucun réseau de surface sur toute l'étendue du bassin karstique.

Dès leur entrée dans le bassin calcaire les rivières vont donc montrer des pertes plus ou moins importantes, ces pertes étant fonction du niveau de la nappe, du débit amont, mais également de la capacité d'absorption du lit des cours d'eau.

La Venelle, qui montre des infiltrations graduelles à partir de Selongey, tant en étiage qu'en crue, se perd totalement par injection dans une gravière sur karst ouvert à l'amont immédiat de Lux. Seules les fortes crues ne peuvent être totalement absorbées et entraînent des inondations périodiques dans la partie Est de l'agglomération de Lux.

Sur l'Ignon, les pertes demeurent minimes entre Issur-Tille et Til-Châtel, le substratum marneux ("Argovien") étant relativement proche.

Sur la Tille, les infiltrations sont sensibles à l'aval immédiat de Crecey-sur-Tille, la rivière pouvant s'assécher périodiquement en étiage au niveau des forges de Til-

LA HOUILLE BLANCHE/N $\mathrm{N}^{\circ} 2-1983$ 


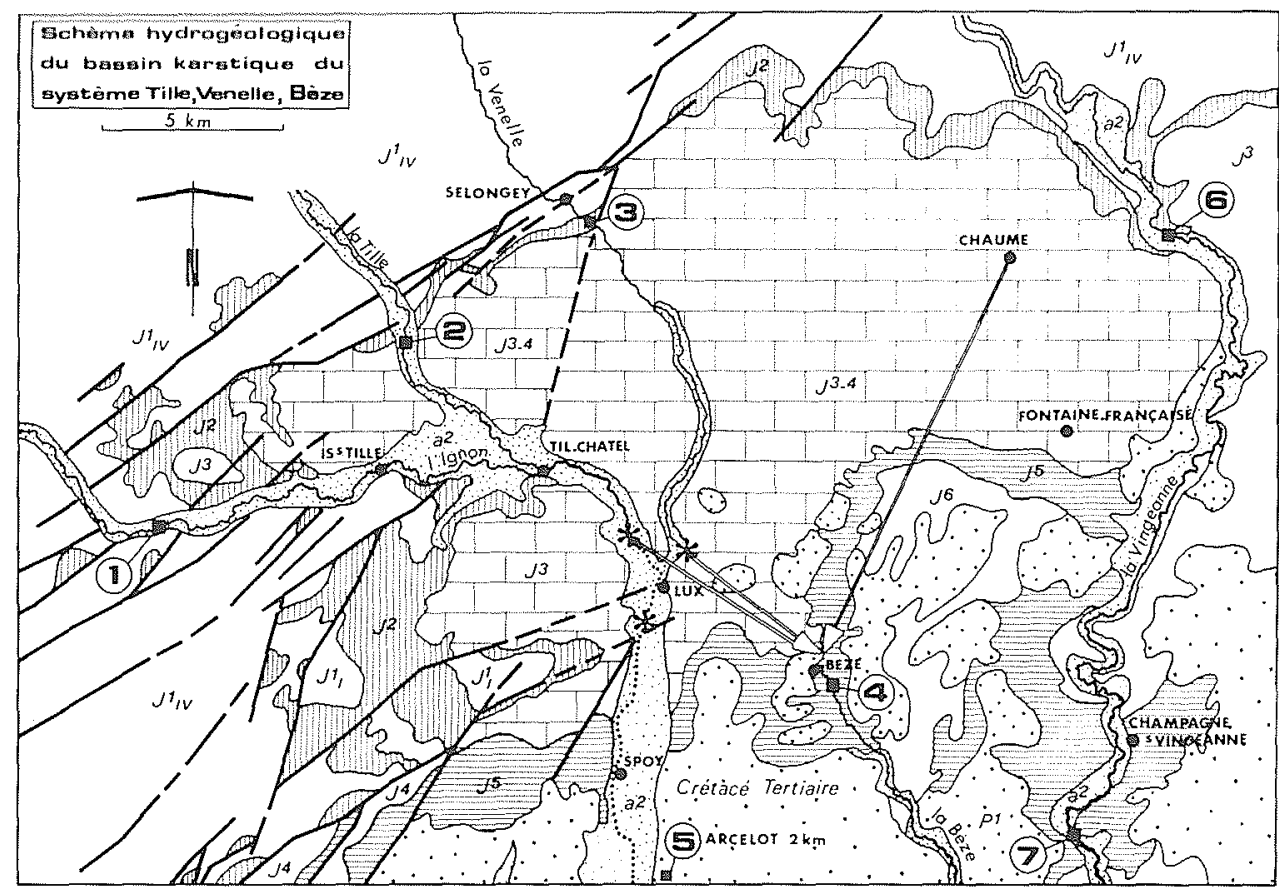

Figure 1 - Cadre géologique. Légende $: a^{2}$ Alluvions de fonds de vallée $; p^{1}$ Formations argileuses plio-quaternaires en placage ; $J^{6}$ Calcaires portlandiens $; J^{5}$ Marnes et calcaires argileux du Kimméridgien sup. (limite aval du bassin karstique) $; J^{3-4}$ Calcaires séquanien et rauraciens (Kimmeridgien inf.-Oxfordien sup.) ; $J^{2}$ Marnes sableuses et calcaires pélitiques argoviens (Oxfordien sup.) (limite amont du bassin karstique) ; $J_{\mathrm{T}}^{1} \mathrm{~V}$ Formations essentiellement calcaires du Jurassique moyen; $\longrightarrow$ Circulation karstique prouvé par coloration ; * Pertes; Station de jaugeage. 1 -Dienay. 2 -Crecey. 3 -Selongey. 4 -Bèze. 5 -Arcelot. 6 -St Maurice-sur-Vingeanne. 7 - Oisilly.

Châtel. Ce n'est qu'entre Beire-le-Châtel et Fouchanges, à $4,5 \mathrm{~km}$ en aval du toit du réservoir calcaire (seuil des marnes du Kimméridgien) que la Tille reprend un écoulement de surface (drainage de la nappe des alluvions de la Tille moyenne).

Différentes colorations, réalisées en 1969 et 1971, au niveau des pertes de la région de Lux ont montré des liaisons Tille-Bèze et Venelle-Bèze avec des vitesses apparentes de propagation comprises entre $100 \mathrm{~m} / \mathrm{h}$ (étiage) et $200 \mathrm{~m} / \mathrm{h}$ (eaux moyennes).

\section{Infrastructure hydrométrique}

Chacune des rivières est équipée, à son entrée dans le bassin karstique, d'une station de jaugeage implantée sensiblement à l'aplomb du seuil marneux amont, les sorties du système étant contrôlées d'une part à Arcelot sur la Tille, d'autre part à Bèze à $1,5 \mathrm{~km}$ en aval de la résurgence (fig. 2).

Les caractéristiques principales de ces stations sont récapitulées dans le tableau I .

Dans le cadre des études entreprises en 1981 pour la protection des ressources en eau de l'ensemble des bassins concernés, trois sections ont également fait l'objet de mesures périodiques sur la Tille entre TilChâtel et Spoy :

- Aval Forges de Til-Châtel

- Lux

- Spoy
Tableau I

\begin{tabular}{|l|l|l|r|r|r|}
\hline $\begin{array}{l}\text { Cours } \\
\text { d'eau }\end{array}$ & Station & $\begin{array}{c}\text { Code } \\
\text { hydrolo- } \\
\text { gique }\end{array}$ & $\begin{array}{c}\text { B.V. } \\
\text { superfi- } \\
\text { ciel } \\
\left(\mathrm{km}^{2}\right)\end{array}$ & $\begin{array}{c}\text { B.V. } \\
\text { Réel } \\
\left(\mathrm{km}^{2}\right)\end{array}$ & $\begin{array}{l}\text { Date de } \\
\text { mise en } \\
\text { service }\end{array}$ \\
\hline Venelle & Selongey & 21110901 & 55,0 & $(55,0)$ & 1970 \\
\hline Tille & $\begin{array}{l}\text { Crecey } \\
\text { S/Tille }\end{array}$ & 21120401 & 234 & $(234)$ & 1970 \\
\hline Ignon & Dienay & 21121502 & 316 & $(316)$ & 1972 \\
\hline Tille & Arcelot & 21122401 & 862 & $735^{*}$ & 1966 \\
\hline Bezze & $\begin{array}{l}\text { Bèze } \\
\text { Pont de } \\
\text { Rome }\end{array}$ & 21111502 & 32,6 & Inconnu & 1981 \\
\hline
\end{tabular}

* Sans la Venelle

\section{Débits caractéristiques d'étiage aux stations de jaugeage}

Dans le cadre de cette note, spécifique aux étiages de la Tille entre Til-Châtel et Spoy, seules seront prises en compte les données des stations de Crecey-sur-Tille et de Diénay.

Les variables considérées sont :

- le débit moyen mensuel minimal annuel (noté QMNA)

- le débit moyen minimal annuel de 10 jours consécutifs (noté VCN 10)

- le débit moyen minimal annuel de 30 jours consécutifs (noté VCN 30). 
Figure 2 - Situation des stations de jaugeage et des points de mesure

(1) Tille à l'aval des Forges de Til-Châtel

(2) Tille à Lux

(3) Tille à Spoy

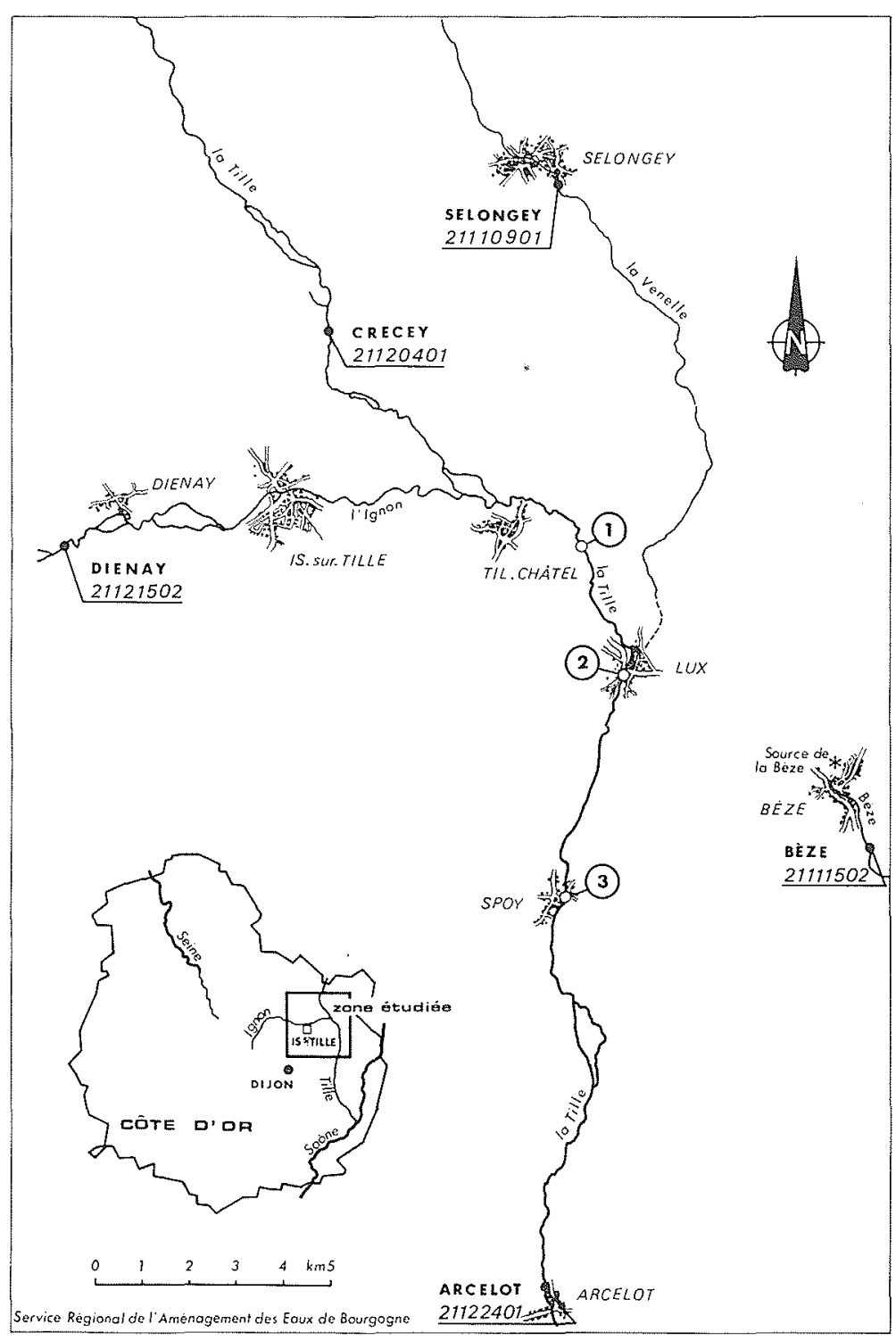

L'étude en cours, sur les hauts bassins de la Tille et de la Venelle, donne comme débits caractéristiques d'étiage, sur les deux stations considérées, les valeurs du tableau II .

Les débits spécifiques d'étiage apparaissent deux fois plus élevés sur l'Ignon que sur la Tille, quelle que soit la variable considérée.

Pour pouvoir transposer éventuellement ces débits à l'aval de la confluence de l'Ignon et de la Tille, il a également été étudié la distribution statistique du QMNA correspondant à la somme des apports des deux stations. Les résultats obtenus (tableau III) sont relativement proches de ceux donnés par la simple addition des débits caractéristiques du tableau II.
Tableau II

\begin{tabular}{|c|c|c|c|c|c|}
\hline \multirow{2}{*}{$\begin{array}{l}\text { Débits } \\
\text { caracté- } \\
\text { ristiques }\end{array}$} & \multirow{2}{*}{$\begin{array}{c}\text { Fré- } \\
\text { quence } \\
\text { de non } \\
\text { dépas- } \\
\text { sement } \\
\end{array}$} & \multicolumn{2}{|c|}{ Ignon à Dienay } & \multicolumn{2}{|c|}{ Tille à Crecey S/Tille } \\
\hline & & $\left(\mathrm{m}^{3} / \mathrm{s}\right)$ & $\begin{array}{c}q \\
\left(1 / \mathrm{s} / \mathrm{km}^{2}\right)\end{array}$ & $\left(\mathrm{m}^{3} / \mathrm{s}\right)$ & $\begin{array}{c}q \\
\left(1 / \mathrm{s} / \mathrm{km}^{2}\right)\end{array}$ \\
\hline \multirow{3}{*}{ QMNA } & $F=0,8$ & 1,24 & 3,9 & 0,450 & 1,9 \\
\hline & $F=0,5$ & 0,680 & 2,2 & 0,220 & 0,9 \\
\hline & $F=0,2$ & 0,370 & 1,2 & 0,110 & 0,5 \\
\hline \multirow{3}{*}{ VCN 30} & $F=0,8$ & 1,15 & 3,6 & 0,400 & 1,7 \\
\hline & $F=0,5$ & 0,620 & 2,0 & 0,200 & 0,9 \\
\hline & $F=0,2$ & 0,340 & 1,1 & 0,090 & 0,4 \\
\hline \multirow{3}{*}{ VCN 10} & $F=0,8$ & 0,990 & 3,1 & 0,340 & 1,5 \\
\hline & $F=0,5$ & 0,350 & 1,7 & 0,160 & 0,7 \\
\hline & $F=0,2$ & 0,280 & 0,9 & 0,070 & 0,3 \\
\hline
\end{tabular}


Tableau III

\begin{tabular}{|c|c|c|c|}
\hline \multirow{2}{*}{$\begin{array}{c}\text { Débits } \\
\text { caractéristiques }\end{array}$} & $\begin{array}{c}\text { Fréquence } \\
\text { de non } \\
\text { dépassement }\end{array}$ & \multicolumn{2}{|c|}{$\begin{array}{c}\text { Débit totat Ignon }+ \text { Tille } \\
\left(B . V .=550 \mathrm{~km}^{2}\right)\end{array}$} \\
\cline { 3 - 4 } & $Q\left(\mathrm{~m}^{3} / \mathrm{s}\right)$ & $q\left(1 / \mathrm{s} / \mathrm{km}^{2}\right)$ \\
\hline \multirow{3}{*}{ QMNA } & $F=0,8$ & 1,70 & 3,1 \\
\cline { 2 - 4 } & $F=0,5$ & 0,920 & 1,7 \\
\cline { 2 - 4 } & $F=0,2$ & 0,500 & 0,9 \\
\hline \multirow{3}{*}{ VCN 30 } & $F=0,8$ & 1,60 & 2,9 \\
\cline { 2 - 4 } & $F=0,5$ & 0,800 & 1,5 \\
\hline \multirow{3}{*}{ VCN 10 } & $F=0,2$ & 0,430 & 0,8 \\
\hline & $F=0,8$ & 1,34 & 2,4 \\
\cline { 2 - 4 } & $F=0,5$ & 0,690 & 1,3 \\
\cline { 2 - 4 } & $F=0,2$ & 0,320 & 0,6 \\
\hline
\end{tabular}

N.B. Les différents ajustements statistiques ont été effectués par la méthode des moments suivant la loi Log-Normale.

\section{Prédétermination des débits d'étiage à l'aval de} la confluence Tille-Ignon

\section{Sections considérées}

Les trois principales sections sur lesquelles ont été. tentées les estimations des débits d'étiage sont :

- La Tille à l'aval des Forges de Til-Châtel sous le pont situé au lieu-dit "En la Cognée", à environ $1 \mathrm{~km}$ en aval des forges. Le bassin versant total à ce niveau atteint $663 \mathrm{~km}^{2}$.

- La Tille au pont de Lux, où le bassin versant superficiel est de $674 \mathrm{~km}^{2}$ (sans la Venelle)

- La Tille au pont de la D 112 à Spoy, qui présente à ce niveau un bassin versant total de $846 \mathrm{~km}^{2}$, mais dont le bassin versant effectif en étiage n'est que de $719 \mathrm{~km}^{2}$ après exclusion de celui de la Venelle $\left(127 \mathrm{~km}^{2}\right)$.

\section{Transposition des débits spécifiques d'étiage définis à partir des stations de jaugeage}

La simple transposition du débit total d'étiage enregistré aux stations, conduirait, pour les débits mensuels minimum annuels (QMNA), aux valeurs du tableau IV.

Or l'observation montre que la Tille s'assèche périodiquement sur chacune de ces sections, pendant des périodes plus ou moins longues suivant l'hydraulicité de l'année considérée (plus de 4 mois à Spoy en 1976 et 1978).
Tableau IV

\begin{tabular}{|c|c|c|c|}
\hline QMNA & $\begin{array}{c}\text { Aval forges } \\
\text { Til-Châtel }\end{array}$ & Lux & Spoy \\
\hline$F=0,8$ & $2,06 \mathrm{~m}^{3} / \mathrm{s}$ & $2,09 \mathrm{~m}^{3} / \mathrm{s}$ & $2,23 \mathrm{~m}^{3 / \mathrm{s}}$ \\
$F=0,5$ & $1,13 \mathrm{~m}^{3} / \mathrm{s}$ & $1,15 \mathrm{~m}^{3} / \mathrm{s}$ & $1,22 \mathrm{~m}^{3 / \mathrm{s}}$ \\
$F=0,2$ & $0,600 \mathrm{~m}^{3} / \mathrm{s}$ & $0,610 \mathrm{~m}^{3} / \mathrm{s}$ & $0,650 \mathrm{~m}^{3 / \mathrm{s}}$ \\
\hline
\end{tabular}

Afin de transposer les débits amont au droit de ces sections, il est donc nécessaire de déterminer, pour chacune d'elle, le seuil au-dessous duquel la rivière peut s'assècher.

\section{Recherche de liaisons entre sections de mesure et sta- tions de jaugeage}

Pour chacune des trois sections considérées, il a été recherché une liaison entre la somme des débits mesurés aux stations de Diénay et Crecey et les débits mesurés au droit de ces sections (fig. 3,4 et 5).

Les débits pris en compte concernant essentiellement l'année 1981, les relations mises en évidence traduisent une co-variation des débits. Il serait nécessaire de disposer de mesures sur plusieurs années pour s'assurer de la stabilité de ces relations.

Pour chacune des trois sections, les relations provisoires s'expriment sous la forme suivante :

- Tille à l'aval des forges de Til-Châtel

$Q i=1,30 \mathrm{Q}$ (Diénay + Crecey) - 0,92(en $\left.\mathrm{m}^{3} / \mathrm{s}\right)$

L'extrapolation de la droite montre que la rivière s'assèche à ce niveau lorsque le débit amont descend audessous de $0,70 \mathrm{~m}^{3} / \mathrm{s}$.

- Tille à Lux

$O i=1,27 \mathrm{Q}$ (Diénay + Crecey) - 1,19 (en $\left.\mathrm{m}^{3} / \mathrm{s}\right)$

L'assèchement à ce niveau semble se produire lorsque le débit total amont descend au-dessous de $0,94 \mathrm{~m}^{3} / \mathrm{s}$.

- Tille à Spoy

$Q i=1,22 \mathrm{Q}$ (Diénay + Crecey) - 1,40 (en $\left.\mathrm{m}^{3} / \mathrm{s}\right)$

L'assèchement au niveau de Spoy se produirait lorsque le débit total enregistré aux stations de Diénay et Crecey s'abaisse au-dessous de $1,15 \mathrm{~m}^{3} / \mathrm{s}$.

Calcul des débits caractéristiques d'étiage à partir des relations entre sections de mesure et stations de jaugeage

A partir des relations définies ci-dessus, et sous réserve de leur stabilité, il est possible d'estimer les ordres de grandeur des débits caractéristiques d'étiage au droit des trois sections considérées. Seul le débit moyen mensuel minimum annuel a été calculé afin de ne pas surcharger le tableau $\mathrm{V}$.

Tableau V

\begin{tabular}{|c|c|c|c|c|c|c|}
\hline \multirow{2}{*}{} & \multicolumn{2}{|c|}{ Aval Til-Châtel } & \multicolumn{2}{c|}{ Lux } & \multicolumn{2}{c|}{ Spoy } \\
\cline { 2 - 7 } & $Q\left(\mathrm{~m}^{3 / \mathrm{s}}\right)$ & $q\left(1 / \mathrm{s} / \mathrm{km}^{2}\right)$ & $Q\left(\mathrm{~m}^{3} / \mathrm{s}\right)$ & $q\left(1 / \mathrm{s} / \mathrm{km}^{2}\right)$ & $Q\left(\mathrm{~m}^{3} / \mathrm{s}\right)$ & $Q\left(1 / \mathrm{s} / \mathrm{km}^{2}\right)$ \\
\hline$F=0,8$ & 1,29 & 1,9 & 0,97 & 1,4 & 0,67 & 0,9 \\
$F=0,5$ & 0,28 & 0,4 & 0 & 0 & 0 & 0 \\
$F=0,2$ & 0 & 0 & 0 & 0 & 0 & 0 \\
\hline
\end{tabular}




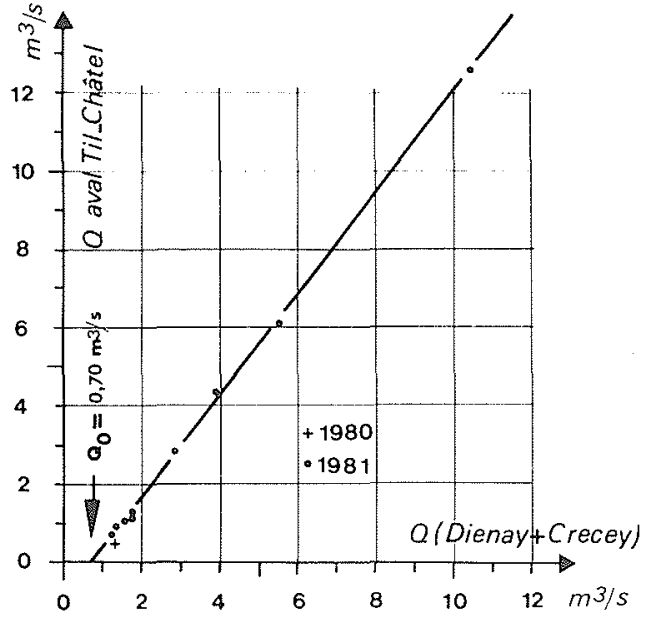

Figure 3 - Relation entre le débit mesuré sur la Tille à l'aval des Forges de Til-Châtel et le débit total mesuré aux stations hydrométriques de Dienay (Ignon) et de Crecey (Tille)

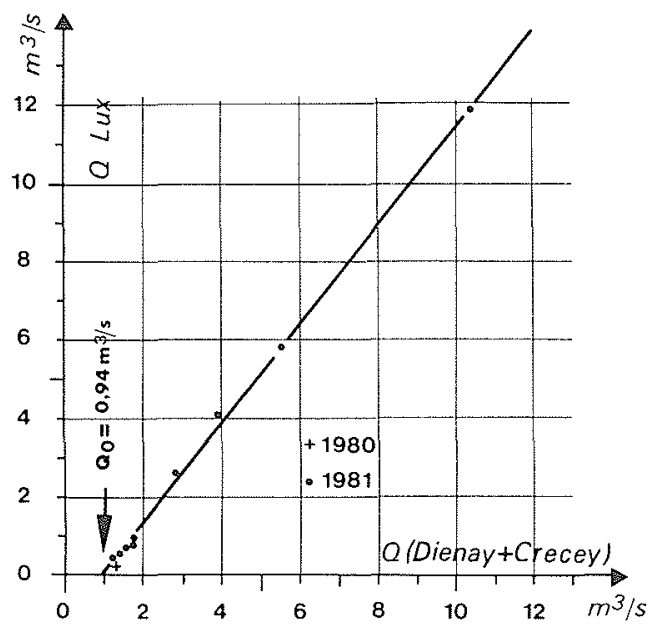

Figure 4 - Relation entre le débit mesuré sur la Tille à Lux et le débit total mesuré aux stations hydrométriques de Dienay (Ignon) et de Crecey (Tille)

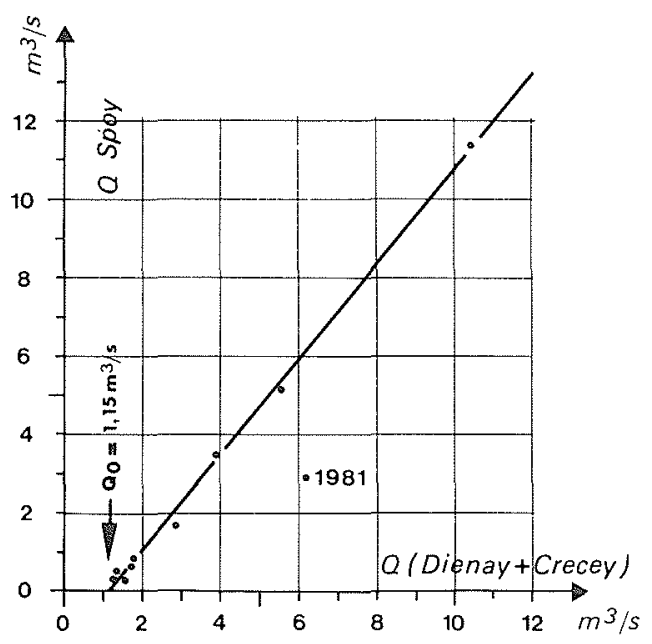

Figure 5 - Relation entre le débit mesuré sur la Tille à Spoy et le débit total mesuré aux stations hydrométriques de Dienay (Ignon) et de Crecey (Tille)

Courbes des débits non dépassés pendant $n$ jours consécutifs pour différentes fréquences
La prise en compte des pertes fait chuter de manière spectaculaire les débits caractéristiques d'étiage. A titre de vérification signalons qu'au cours de la période 1972-1981 (10 ans), la Tille au niveau de Spoy est restée pérenne uniquement en 1977 et 1981 ( 2 années sur 10), et qu'elle s'est asséchée pendant plus d'un mois en 1972, 1974, 1976 et 1978 (soit 4 années sur 10).

\section{Estimation des durées d'assèchement}

Les durées d'assèchement dépendant du nombre de jours consécutifs pendant lequel le débit descend audessous du seuil correspondant à la capacité d'absorption du tronçon de rivière considéré, nous avons établi les courbes des débits non dépassés pendant $n$ jours consécutifs pour différentes fréquences (débits notés QCNn)

A titre d'exemple, un débit amont de $1 \mathrm{~m}^{3} / \mathrm{s}$ (total de l'Ignon à Diénay et de la Tille à Crecey) n'est pas dé. passé 20 jours par an, une année sur deux. (fig. 6).

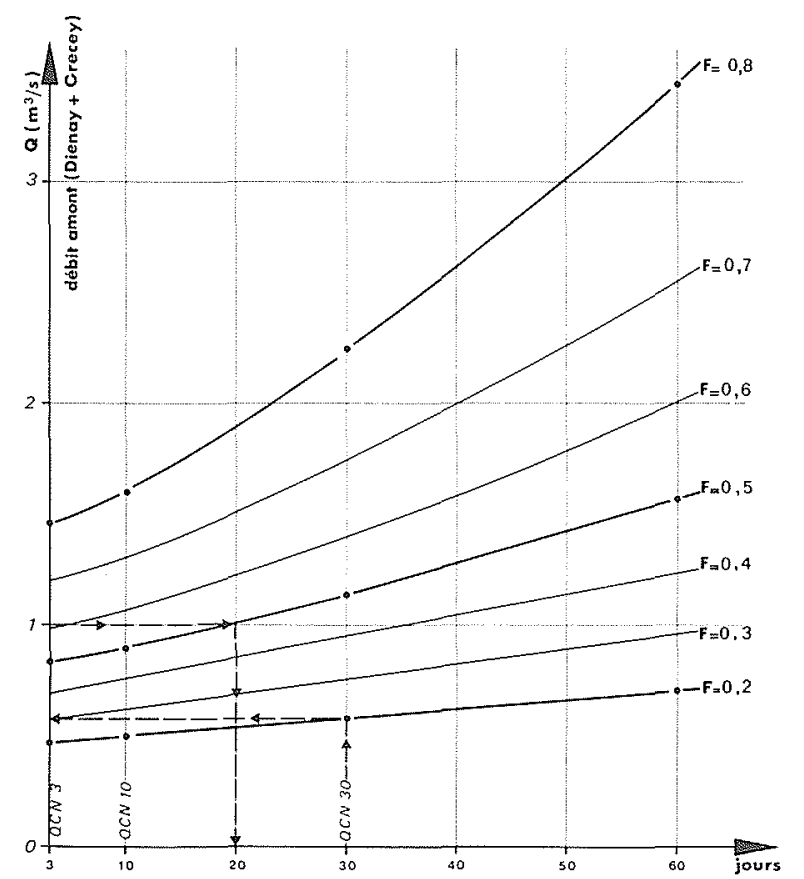

Figure 6 - Débit total de la Tille et de l'Ignon en amont de TilChâtel-Stations de jaugeage de Dienay et de Crécey. Courbes des débits non dépassés pendant $n$ jours consécutifs (QCNn) pour différentes fréquences.

En traçant sur ce graphe une ligne parallèle à l'axe des abscisses, et passant par le point d'ordonnée correspondant au débit amont en-dessous duquel la rivière s'assèche, il est possible d'estimer les durées d'assèchement au droit de chacune des sections. Cette ligne correspond également à l'origine de l'axe d'ordonnée porté sur la droite du graphe, et qui donne directement le débit au droit de la section considérée (fig. 7, 8 et 9).

L'assèchement commençant par la section la plus aval et se propageant vers l'amont au fur et à mesure de la diminution des apports, nous allons définir les principales caractéristiques des étiages d'aval en amont. 


\section{La Tille à Spoy (fig. 7)}

Par lecture directe sur l'axe des abscisses passant par $1,15 \mathrm{~m}^{3} / \mathrm{s}$ (débit amont au-dessous duquel la Tille s'assèche à Spoy), les durées caractéristiques d'assèchement de la Tille à ce niveau seraient :

- assèchement durant 3 jours consécutifs, 7 années sur 10 - assèchement durant 15 jours consécutifs, 6 années sur 10

- assèchement durant 30 jours consécutifs, 5 années sur 10

- assèchement durant 50 jours consécutifs, 4 années sur 10

A titre de vérification, les observations faites par le S.R.A.E. Bourgogne sur la Tille à Spoy montrent les durées d'assèchement suivantes (à quelques jours près) :

$$
\begin{array}{lll}
-1972: 50 \text { jours } & -1977: \text { néant } \\
-1973: 15 \text { jours } & -1978: 125 \text { jours } \\
-1974: 45 \text { jours } & -1979: 15 \text { jours } \\
-1975: 20 \text { jours } & -1980: 8 \text { jours } \\
-1976: 130 \text { jours } & -1981: \text { néant }
\end{array}
$$

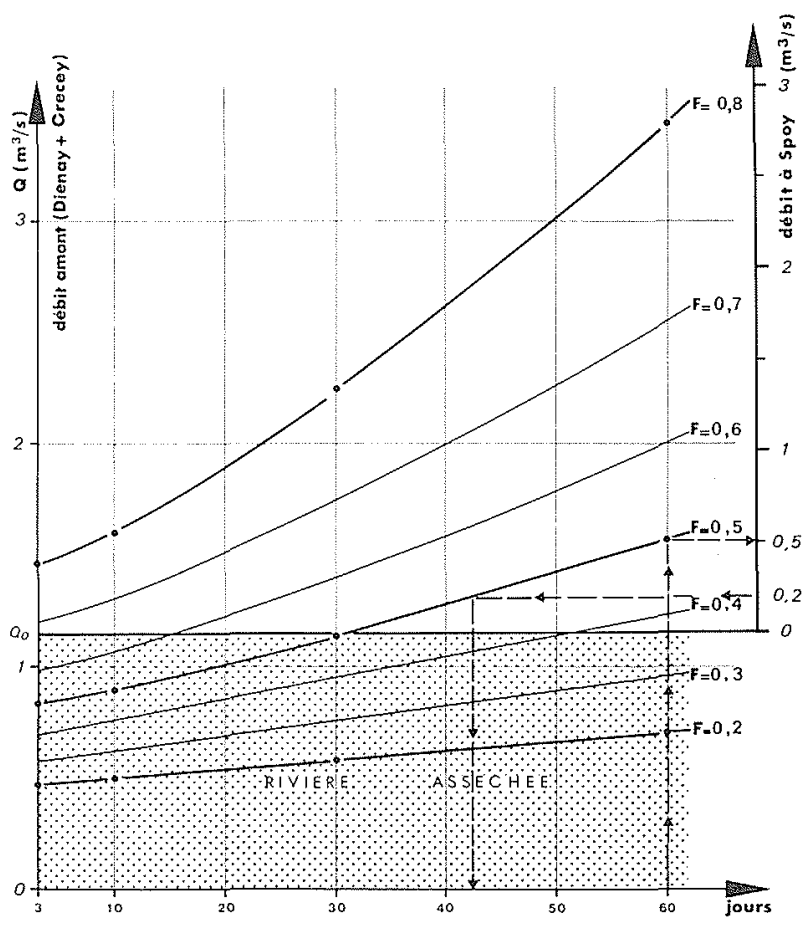

Figure 7 - Débit de la Tille à Spoy - Section de jaugeage $n^{\circ} 3$ Courbes des débits non dépassés pendant $n$ joưrs consécutifs $(\mathrm{QCNn})$ pour différentes fréquences)

D'autre part, l'axe des ordonnées porté sur la partie droite du graphe permet de connaître la valeur audessous de laquelle le débit va descendre durant $n$ jours consécutifs.

\section{Exemples :}

- un débit de $200 \mathrm{l} / \mathrm{s}$ n'est pas dépassé durant environ 40 jours consécutifs une année sur deux

- le débit au-dessous duquel la Tille peut se maintenir une année sur deux durant 2 mois est de $500 \mathrm{l} / \mathrm{s}$.

\section{La Tille à Lux (fig. 8)}

D'après le graphe, les durées caractéristiques d'assèchement de la Tille à Lux seraient :

- assèchement durant 3 jours consécutifs, 6 années sur 10

- assèchement durant 15 jours consécutifs, 5 années sur 10

- assèchement durant 30 jours consécutifs, 4 années sur 10

- assèchement durant 55 jours consécutifs, 3 années sur 10

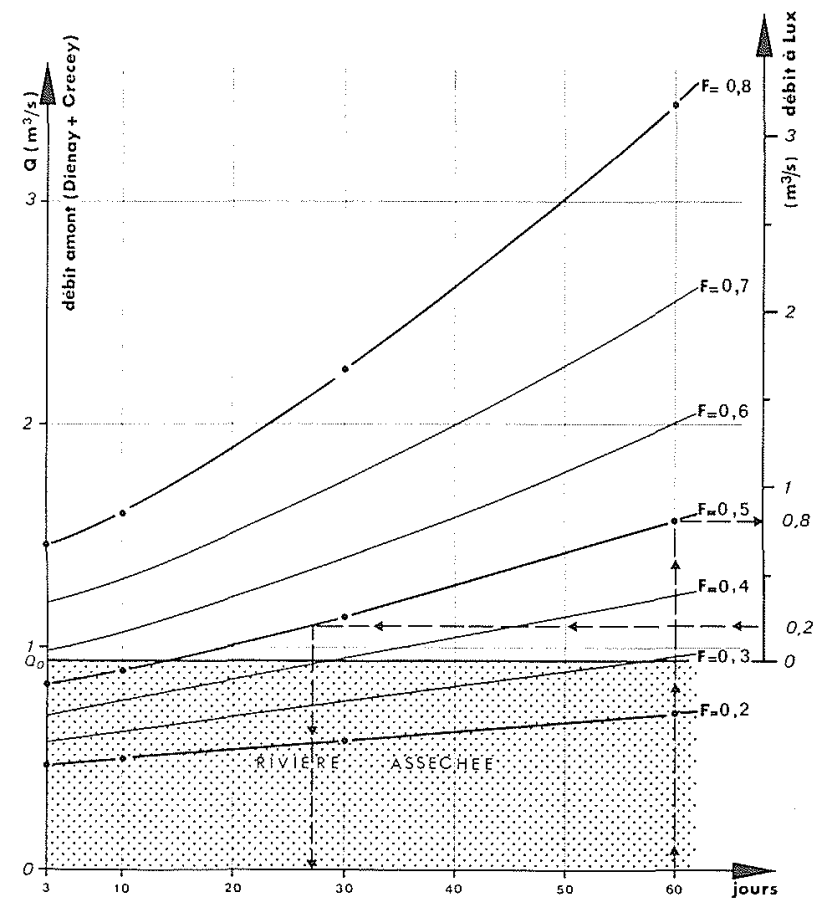

Figure 8. - Débit de la Tille à Lux - Section de jaugeage $\mathrm{n}^{\circ} 2$ Courbes des débits non dépassés pendant $n$ jours consécutifs (QCNn) pour différentes fréquences.

Comme dans le cas précédent, il est possible d'estimer le seuil au-dessous duquel le débit peut se maintenir, durant $n$ jours consécutifs :

- le débit de $200 \mathrm{l} / \mathrm{s}$ n'est pas dépassé durant environ 30 jours consécutifs, une année sur deux

- le débit au-dessous duquel la Tille peut se maintenir une année sur deux durant 2 mois est de $800 \mathrm{l} / \mathrm{s}$.

\section{La Tille à l'aval des forges de Til-Châtel (fig. 9).}

Les durées caractéristiques d'assèchement de la Tille à Til-Châtel seraient :

- assèchement durant 3 jours consécutifs, 4 années sur 10

- assèchement durant 20 jours consécutifs, 3 années sur 10

- assèchement durant 60 jours consécutifs, 2 années sur 10

A titre d'exemple, le débit de $200 \mathrm{l} / \mathrm{s}$ n'est pas dépassé 6 jours consécutifs, une année sur deux. De même le débit au-dessous duquel la Tille peut se maintenir une année sur deux durant 60 jours est de $1,1 \mathrm{~m}^{3} / \mathrm{s}$. 


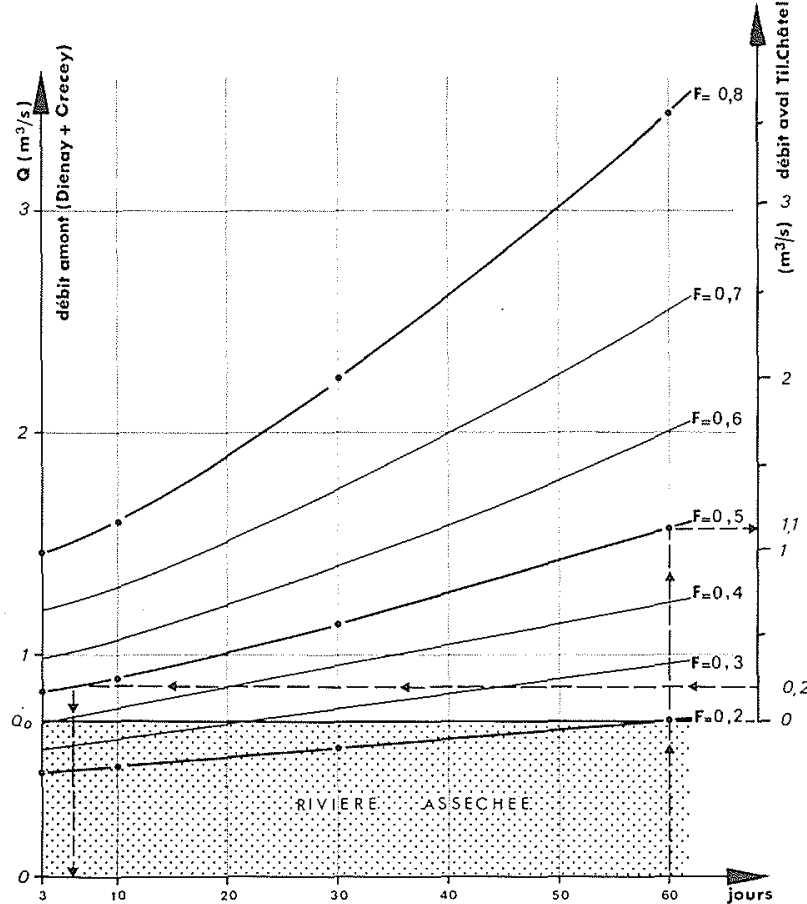

Figure 9 - Débit de la Tille en aval des forges de Til-ChâtelSection de jaugeage $n^{\circ} 1$. Courbes des débits non dépassés pendant $n$ jours consécutifs (QCNn) pour différentes fréquences.

Il est à signaler que les observations, faites au niveau des forges de Til-Châtel à $1 \mathrm{~km}$ en amont de ce site, confirment ces estimations. Au cours des 40 dernières années, la Tille se serait asséchée en aval immédiat des forges pratiquement une année sur deux, les durées d'assèchement ayant atteint ou dépassé 4 mois en 1976 et 1947 (communication orale de M. Perrin Forges de Til-Châtel).

\section{Remarques importantes}

Les différents abaques élaborés (fig. 7, 8 et 9) sont avant tout destinés à fixer les ordres de grandeur des durées d'assèchement et des débits non dépassés pendant $n$ jours consécutifs, notamment pour les raisons suivantes:

- la série observée aux stations de Diénay et Crecey est de trop courte durée ( 9 années communes seulement) pour estimer avec une précision suffisante les périodes de retour des débits d'étiage

- les seuils au-dessous desquels la rivière s'assèche n'ont été déterminés que sur la base des mesures effectuées en 1980 et 1981 . Une modification minime du seuil de l'ordre de 10 à $20 \%$ peut entraîner une modification importante des durées d'assèchement, les courbes étant très aplaties dans la partie inférieure des graphes

- la gamme des faibles débits est celle où l'erreur relative commise est la plus importante.

\section{Conclusion}

Dans les secteurs de pertes des cours d'eau en milieu calcaire, la simple transposition des débits spécifiques d'étiage, déterminés sur les stations les plus proches, ne permet pas une estimation correcte des débits.

Seules des mesures, en nombre suffisant, réparties sur plusieurs années, peuvent permettre, par des relations simples, la transposition des débits au droit des sections non équipées en station de jaugeage. Si les relations demeurent stables d'une année à l'autre, il est alors possible d'estimer le seuil au-dessous duquel la rivière peut s'assécher.

Parmi les sections envisagées dans le cadre de cette note, celle située ă l'aval de Til-Châtel montrerait les débits d'étiage suivants :

- par transposition directe des caractêristiques définies au droit des stations de jaugeage, le débit moyen mensuel d'étiage (QMNA) de fréquence quinquennale sèche atteindrait $600 \mathrm{l} / \mathrm{s}$

- la relation définie entre cette section et le débit total mesuré au niveau des stations de jaugeage montre en fait que ce même débit caractéristique est égal à 0 , et que la rivière peut s'assécher à ce niveau durant 60 jours consécutifs, 2 années sur 10.

Tout projet de station d'épuration dans ce secteur devra donc tenir compte de cette caractéristique, d'autant plus que les eaux infiltrées rejoignent ensuite la résurgence karstique de la Bèze captée pour l'A.E.P.

\section{Bibliographie}

\section{A - Méthodologie}

R. HLAVEK (1967). - L'étude hydrologique des étiages (ENGREF, $2^{\mathrm{e}}$ session de recyclage - Juin 1967)

R. HLAVEK (1967), - Etiages et besoins en eau (Ministère de l'Agriculture, stage de Strasbourg.Octobre 1967)

D. DUBAND et P. GUILLOT (1967). - Calcul de la probabilité d'étiage prolongé à l'aide de corrélations entre pluies et débits (Symp, international d'Hydrologie - Fort Collins sept. 1967)

G.R. OBERLIN, G.C. GALEA et J.T. TONI (1973). - Intérêt des jaugeages épisodiques pour l'estimation des étiages de petits bassins non équipés (Cah. ORSTOM, ser. Hydrol., vol. $\left.X, N^{\circ} 4,1973\right)$

ANONYME (1978). - Distribution de frëquence adaptée aux étiages - Méthodes manuelles et graphiques (Note technique $N^{\circ} 6$ CTGREF Juillet 1978)

ANONYME (1979). - Etude fréquentielle des étiages (CTGREF, Informations techniques, cahier 33 , note $\mathrm{N}^{\circ} 5$ mars 1979)

G.R. OBERLIN (1982). - Assessment of low water characteristics on unobserved basins of the eastern region of Paris with use of representative basin data (UNESCO, Studies and reports in Hydrologie, $\mathrm{N}^{\circ} 54$ )

\section{$B$ - Connaissance du bassin karstique des sources de la Bèze}

B. DE LORIOL (1955). - Contribution aux recherches sur l'origne de la Bèze. Sous le Plancher, $\mathrm{N}^{\circ} 61955$, p.p. 1014.

J. DAVID ET R. LIORET (1955). - Les cavités de la Forêt de Velours - Compte-rendu des prospections - Années 1953-1954. Sous le Plancher, $\mathrm{N}^{\circ} 1 / 1955, \mathrm{pp} .8-11$ et $\mathrm{N}^{\circ} 2 /$ 1955 pp. 10-18.

J. MARILLIER (1958). - Bèze - Son histoire, son site, sa grotte - I. Survol d'une histoire de Bèze. Sous le Plancher, $\mathrm{N}^{\circ} 2-1958$, pp. 35-40.

R. VELARD (1958). - Bèze - Son histoire, son site, sa grotte - II. Découverte de la rivière souterraine. Sous le Plancher $\mathrm{N}^{\circ} 3,1958$, pp. 52-57. 
H. TINTANT (1958). - La grotte de Bèze - Géologie et hydrologie. Sous le Plancher, $\mathrm{N}^{\circ} 4 / 5$ - 1958, pp. 68-73.

E. ALESSANDRELLO, M. AMIOT, J.H. DELANCE (1971). Restitution de colorant par vagues successives - Rivière souterraine de Bèze. (21). Spelunca, mém. $\mathrm{N}^{\circ} 7$, pp. 49-59.

E. ALESSANDRELLO, M. AMIOT, A. CLAIR, J. PANIER, P. RAT (1971). - Hydrologie de surface et circulations karstiques dans le systeme Tille-Venelle-Bèze (21). Premiers résultats et vroblemes posés. Actes du colloque d'hydrologie en pays calcaire - Besançon 8 et 9/10/1971, pp. 157-164.

M. AMIOT (1973). - Etude hydrogéologique du bassin de la Haute Vallée des Tilles. Les circulations souterraines dans les calcaires oxfordiens et kimméridgiens au Nord de Dijon. Rapp. inéd. Institut des Sciences de la Terre/S.R.A.E. Bourgogne.

M. AMIOT (1973). - Etude hydrogéologique des calcaires karstiques du Jurassique supérieur de la Haute vallée des Tilles. Le sondage de Fontaine Française. Rapp. inéd. Inst. des Sciences de la Terre/DDA Côte d'Or.

A. PASCAL (1973). - Etude hydrogéologique du bassin de la Haute Vallée des Tilles - Etude lithologique du Jurassique supérieur des bassins de la Tille et de la Venelle. Rapp. inéd. Institut des Sciences de la Terre/S.R.A.E. Bourgogne.

P. GARDAINE et P. LAUREAU (1974). - La rivière souterraine de Bèze - Historique des découvertes - La résurgence de Bèze. Sous le Plancher - Tome 11, fasc. 3-4 pp. 51-77.
J.L. SIMON (1978). - Contribution à l'étude de la dénitrification des eaux sur le parcours surface-nappe phréatique dans un bassin karstique de la région de Dijon. Thèse de doctorat de $3^{\mathrm{e}}$ cycle -. Université de Dijon.

Y. LEMOINE et E.SIWERTZ (1979). - Etude hydrogéologique des calcaires en rive gauche de la Tille. Rapp. inéd. CPGF $\mathrm{N}^{\circ} 1811$ - DDA Côte d'Or.

M. MARION (1980). - Les phénomènes de remontée des niveaux d'eau souterraine dans la région de Lux (21). Etude fréquentielle des cotes maximales. Rapp. inéd. SRAE Bourgogne.

M. MARION (1980). - Piézometre de Spoy (21) - Evolution du niveau piézométrique au cours de la période 1971-1980Essai de prévision de l'étiage 1980. Rapp. inéd. SRAE Bourgogne.

M. MARION (1981). - Estimation des débits de la Bèze du ler au 31 août 1977 et du $1^{\text {er }}$ au 15 février 1980. Rapp. inéd. SRAE Bourgogne.

M. MARION (1981). - Les débits de la Bèze - Note relative à l'estimation provisoire des apports moyens Rapp. inéd. SRAE Bourgogne.

M. MARION (1982). - Protection de l'agglomération de Lux (21) contre les inondations. Etude des crues de la Venelle. Rapp. inéd. S.R.A.E. Bourgogne. 